\title{
Selective Attention to Affective Value Alters How the Brain Processes Olfactory Stimuli
}

\author{
Edmund T. Rolls', Fabian Grabenhorst ${ }^{1}$, Christian Margot ${ }^{2}$, \\ Maria A. A. P. da Silva ${ }^{3}$, and Maria Ines Velazco ${ }^{2}$
}

\begin{abstract}
How does selective attention to affect influence sensory processing? In a functional magnetic resonance imaging investigation, when subjects were instructed to remember and rate the pleasantness of a jasmin odor, activations were greater in the medial orbitofrontal and pregenual cingulate cortex than when subjects were instructed to remember and rate the intensity of the odor. When the subjects were instructed to remember and rate the intensity, activations were greater in the inferior frontal gyrus. These top-down effects occurred not only during odor delivery but started in a preparation period after the instruction before odor delivery, and continued after termination of the odor in a short-term memory period. Thus, depending on the context in which
\end{abstract}

\section{INTRODUCTION}

The primary olfactory areas such as the pyriform cortex project to the orbitofrontal cortex (Ongur \& Price, 2000; Carmichael, Clugnet, \& Price, 1994), in which many neurons respond to odor stimuli based on their reward value (Critchley \& Rolls, 1996a, 1996b; Rolls, Critchley, Mason, \& Wakeman, 1996). In humans, functional magnetic resonance imaging (fMRI) studies of olfaction have shown activations of the pyriform cortex and the orbitofrontal cortex (Plailly, Radnovich, Sabri, Royet, \& Kareken, 2007; Gottfried, Winston, \& Dolan, 2006; Li, Luxenberg, Parrish, \& Gottfried, 2006; de Araujo, Rolls, Velazco, Margot, \& Cayeux, 2005; Small, Gerber, Mak, \& Hummel, 2005; Winston, Gottfried, Kilner, \& Dolan, 2005; Anderson et al., 2003; Rolls, Kringelbach, \& de Araujo, 2003; Royet, Plailly, Delon-Martin, Kareken, \& Segebarth, 2003; Poellinger et al., 2001; Savic, Berglund, Gulyas, \& Roland, 2001; Zald \& Pardo, 2000; Zatorre, Jones-Gotman, \& Rouby, 2000). In particular, it has been shown that activations of the medial orbitofrontal cortex are correlated with the subjective pleasantness of olfactory stimuli, and of the lateral orbitofrontal cortex with unpleasantness, whereas activations of the pyriform cortex are correlated with the intensity but

\footnotetext{
${ }^{1}$ University of Oxford, UK, ${ }^{2}$ Firmenich SA, Geneva, Switzerland,

${ }^{3}$ Campinas State University, Campinas, SP, Brazil
}

odors are presented and whether affect is relevant, the brain prepares itself, responds to, and remembers an odor differently. These findings show that when attention is paid to affective value, the brain systems engaged to prepare for, represent, and remember a sensory stimulus are different from those engaged when attention is directed to the physical properties of a stimulus such as its intensity. This differential biasing of brain regions engaged in processing a sensory stimulus depending on whether the cognitive demand is for affect-related versus more sensory-related processing may be an important aspect of cognition and attention. This has many implications for understanding the effects not only of olfactory but also of other sensory stimuli. not the pleasantness of olfactory stimuli (Rolls et al., 2003). Consistently, another study found that activations in the orbitofrontal cortex were related to pleasantness, and of the pyriform cortex to intensity (Anderson et al., 2003). These studies did not investigate effects of attention on olfactory processing. One study has shown that if attention was being paid to an odor versus no attention to the odor, then more activation to the odor was found in the pyriform cortex (Zelano et al., 2005). However, given that there are separate representations of different attributes of odors, including their intensity, pleasantness, and unpleasantness, it could be that selective attention within the olfactory modality to pleasantness might engage processing in some olfactory areas, and to intensity might activate other cortical areas. This could have very important implications, for how we respond to odors, and potentially other stimuli, too, might depend on the instructions or the context in which the odor was delivered. In this investigation, we investigated this hypothesis.

Moreover, given that top-down attention involves a modulation, probably by biased competition, of the responses of neurons to the incoming sensory stimuli (Rolls, 2008; Deco \& Rolls, 2005a; Rolls \& Deco, 2002; Desimone \& Duncan, 1995), it could be that, before the odor is delivered, but after the instruction to pay attention to pleasantness versus intensity, effects that reflect the topdown input will be evident in brain regions involved in 
attention. This has not been investigated before for topdown modulation of affective versus intensity processing for odor, or indeed for any sensory modality. This is another of the hypotheses we investigated. It is known, however, that top-down attention-related effects can, as would be expected theoretically in view of their biasing effects (Rolls, 2008; Deco \& Rolls, 2005a, 2005b; Rolls \& Deco, 2002; Desimone \& Duncan, 1995), alter activity measured neuronally and with functional neuroimaging before the sensory input is received that is being modulated by attention (McMains, Fehd, Emmanouil, \& Kastner, 2007; Giesbrecht, Weissman, Woldorff, \& Mangun, 2006; Chawla, Rees, \& Friston, 1999; Desimone \& Duncan, 1995).

Further, if there is a representation in the cortex of a stimulus or event, then that cortical area is a strong candidate for the area that maintains a short-term memory representation of the stimulus, by maintained firing of the neurons (Fuster, 2001; Goldman-Rakic, 1996), because of the associatively modifiable cortical recurrent collateral connections between nearby cortical pyramidal cells (Rolls, 2008). In the present context, the hy- pothesis is that parts of the cortex that represent the pleasantness of an odor may be involved in maintaining the pleasantness of an odor in short-term memory, whereas parts of the brain involved in representing the intensity of an odor may be involved in maintaining the intensity of the odor in short-term memory. We investigated this hypothesis too with the experimental design described next.

\section{METHODS}

\section{Overall Design}

The hypotheses raised in the Introduction were investigated with the following task in an fMRI investigation. The identical odor, jasmin, was used in both trial types. A trial started at time $t=0 \mathrm{sec}$ with the visual instruction, either "remember and rate pleasantness" or "remember and rate intensity," shown for $5 \mathrm{sec}$ (the task timing is indicated in Figures 1-4). The differences between the activations in this period were a measure
Figure 1. Attention period: effect of preparing to rate an odor for pleasantness versus intensity. (A) A significant difference was found in the medial orbitofrontal cortex (see cursor location) at [4 38 -28], $z=2.75, p=.038$, and this region extended dorsally into the pregenual cingulate cortex (see further text with respect to this and other figures, where the peak activations are described, and are, for example, for this region, $z=4.66$ ). (B) The time course of the activations at this location for the condition of paying attention to pleasantness versus intensity (mean \pm SEM across subjects). (The peaks of the activations were significantly different: $t=6.7, d f=12, p \ll .001$.) The odor, jasmin, was identical on all trials. The labels show the preparatory period in which the instruction about what to remember and rate on that trial was given ( $t=0$ to $5 \mathrm{sec}$, "attention"); the period in which the odor, jasmin, was delivered ( $t=5$ to $9 \mathrm{sec}$, "odor"); and the period in which the ratings were given (14-18 sec, "ratings"). Some delay is expected after the onset of a stimulus before the BOLD signal changes, and this delay, captured by the hemodynamic response function not used to generate these time courses, peaks at $5-6 \mathrm{sec}$ after the onset. The first value plotted in the time course, at $-1 \mathrm{sec}$, is the value obtained in the period at $-2 \mathrm{sec}$ to 0 sec, that is, the 2-sec period before the instruction labels appeared.

\section{A}

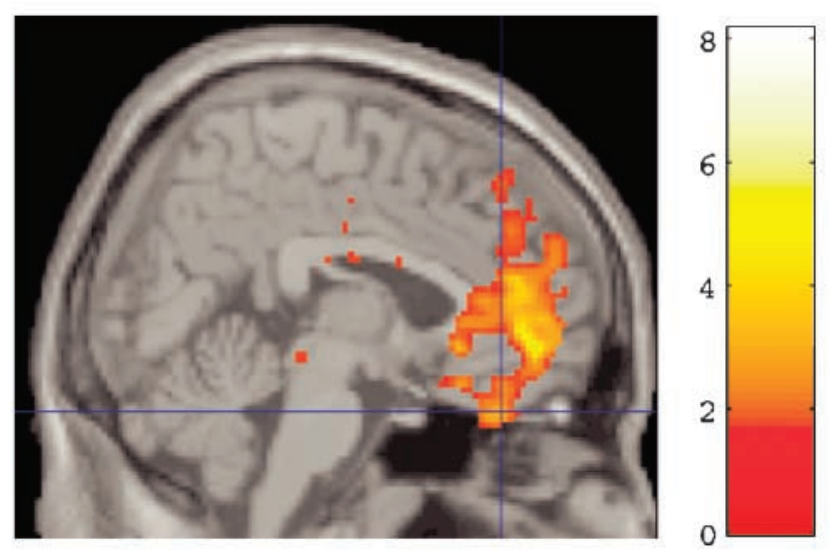

B

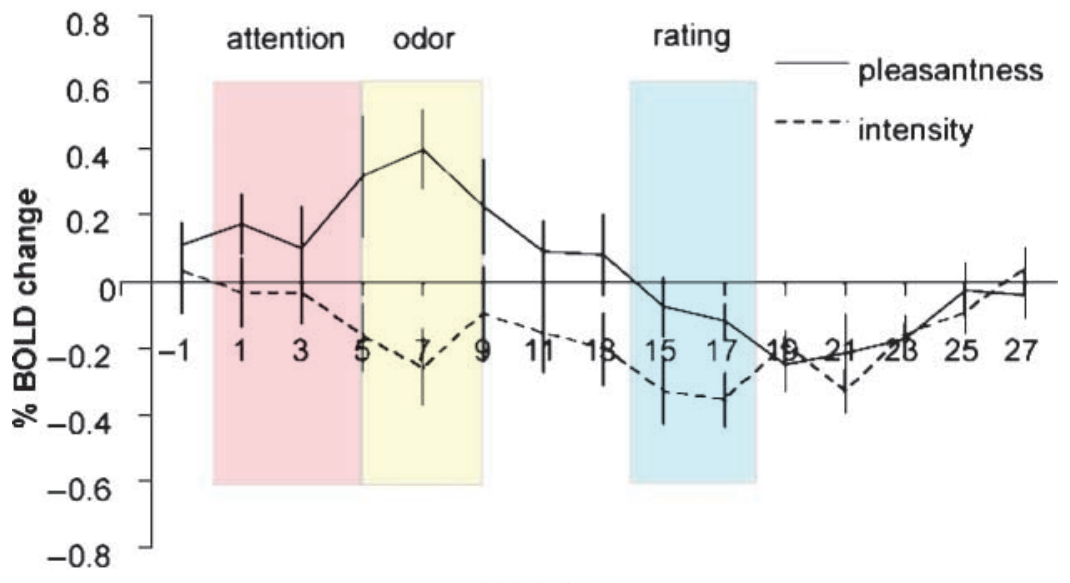

Time (sec) 
Figure 2. Attention period: effect of preparing to rate an odor for intensity versus pleasantness. (A) A significant difference was found in the left inferior frontal gyrus at $[-46$ 248 ], $z=2.66, p<.05$. (B) The time courses of the activations at this location for the conditions of paying attention to intensity or to pleasantness (mean \pm SEM across subjects). (The peaks of the activations were significantly different: $t=5.9$, $d f=12, p \ll .001$.) The odor, jasmin, was identical on all trials. Conventions as in Figure 1.
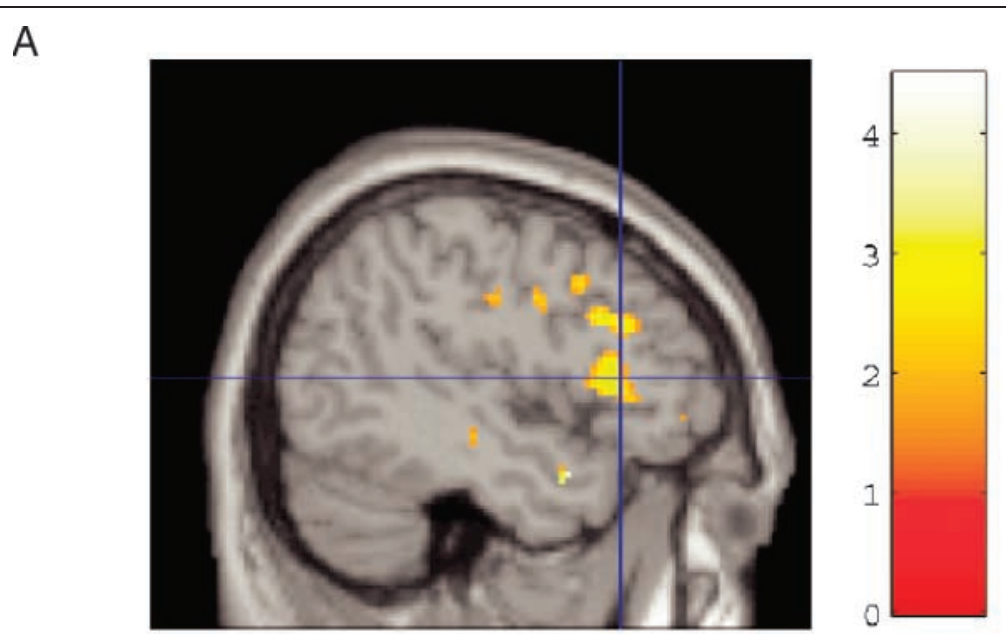

B

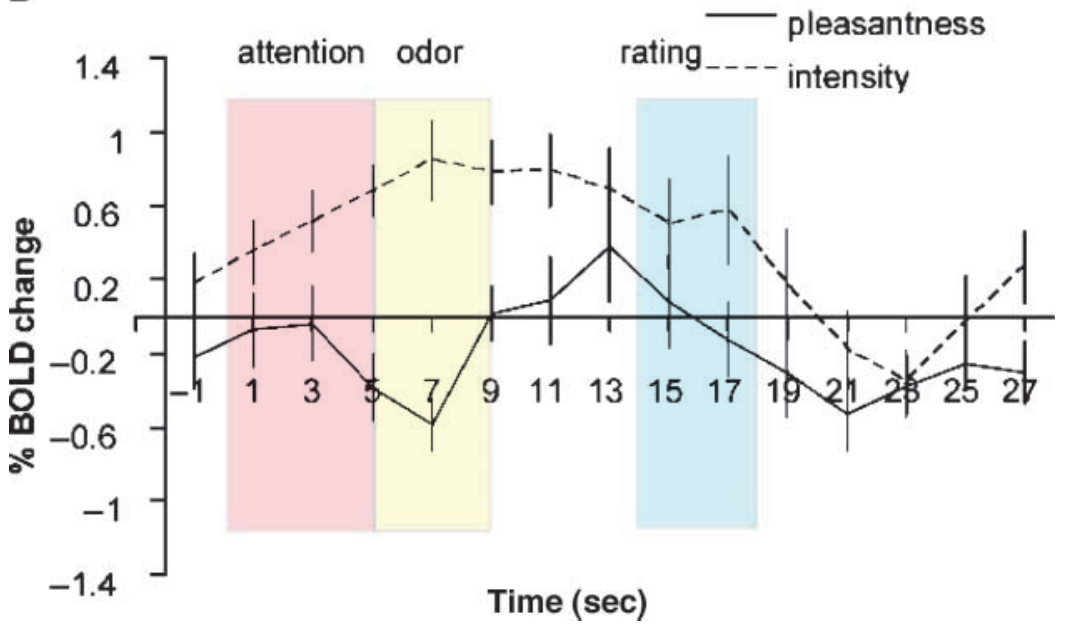

of pre-odor delivery effects of the top-down selective attention instructions. The odor was delivered from $t=$ $5 \mathrm{sec}$ until $t=9 \mathrm{sec}$. The differences between the activations in this period were a measure of the top-down selective attention instructions while the odor was being delivered. After the end of the odor delivery period, the visual instruction was turned off and there was a shortterm memory delay period from $t=9 \mathrm{sec}$ until $t=$ $14 \mathrm{sec}$, in which the pleasantness or the intensity had to be remembered. The activations in this period indicated whether there was a top-down selective attention effect on the short-term memory systems involved in the short-term memory for these different attributes of the odor. The ratings of pleasantness or intensity were made at $t=14$ until $t=18 \mathrm{sec}$ using button-press operated visual rating scales as described previously (Rolls et al., 2003). After this, the odor limonene was delivered for $1 \mathrm{sec}$ (to act as an intertrial dishabituator), and there was then an intertrial interval of 8 sec.

These two trial types, in which the instructions were to remember and rate either pleasantness or intensity, were those most crucial to the present investigation and its hypotheses, and were the first type of analysis that was performed. It was possible to perform a second type of analysis to reveal the effects of top-down attention on activations in the odor delivery period as follows. The above trial types were interspersed in random permuted sequence with other trials that were part of a different investigation in which there was no pretrial instruction or attention period, and the odors used were the same jasmin, 6\% indole (which the subjects rated as unpleasant), a mixture of the two, or clean air, followed by pleasantness and intensity ratings (Grabenhorst, Rolls, Margot, da Silva, \& Velazco, 2007). These other trials were helpful in the current investigation because they enabled brain regions where activations were correlated with the pleasantness of the olfactory stimuli, or their intensity, to be localized. These other trials were also interesting as a comparison for the effects during odor delivery when there had been no pretrial instruction to remember and rate either pleasantness or intensity. On these other trials, the odor was delivered at $t=0$ for $4 \mathrm{sec}$, and at $t=8 \mathrm{sec}$ after trial onset, a pleasantness and then an intensity rating were made, with full details provided by Grabenhorst et al. (2007). In particular, the contrast for the odor delivery period of the trials 
with the instruction "remember and rate pleasantness"these other trials on which there was no explicit instruction to pay attention to only pleasantness or intensity, allowed effects of attention to pleasantness to be estimated. On the other hand, the contrast for the odor delivery period of the trials with the instruction "remember and rate intensity" - these other trials on which there was no explicit instruction to pay attention to only pleasantness or intensity, allowed effects of attention to intensity to be estimated. Clean air was delivered continuously whenever an odor was not being delivered by the olfactometer so that there were no flow rate changes. Each of the six trial types was presented in random permuted sequence nine times. This general protocol and design has been used successfully in previous studies to investigate olfactory cortical areas (de Araujo et al., 2005; Rolls et al., 2003). As six trial types were being run in the scanner at the same time, and included different stimuli, namely indole, a mixture of jasmine and indole which was for the subjects more similar to the jasmine than the indole though not identical to the jasmine, and clean air, it was not evident to the subjects that the stimuli were, in fact, identical on the "remember and rate intensity" and "remember and rate pleasantness" trials. Moreover, no instruction was given to them about exactly what the odors were, let alone that the odor on the "remember and rate intensity" and "remember and rate pleasantness" trials was the same. The procedures made it difficult to expect or recall a particular odor in the attention period of the "remember and rate intensity" and "remember and rate pleasantness" trials and, in any case, the effects described here cannot be related just to expectation or recall of a particular odor because different effects were found on these two trial types.

\section{Participants}

Thirteen healthy volunteers ( 6 men and 7 women, mean age $=26$ years $)$ participated in the study. Ethical approval (Central Oxford Research Ethics Committee) and written informed consent from all subjects were obtained before the experiment.

\section{Stimuli}

A chemically defined jasmin-like model odor ("jasmin") was made that contained the following: benzyl acetate,
Figure 3. Odor period: effect of paying attention to pleasantness versus intensity. (A) A significant difference during the odor period was found in the hypothalamus at [0 -6 -8], $z=2.89, p<.05$. (B) The time courses of the activations at this location for the conditions of paying attention to pleasantness or to intensity (mean \pm SEM across subjects). (The peaks of the activations were significantly different: $t=4.0, d f=12$, $p=.002$.) The odor, jasmin, was identical on all trials. Conventions as in Figure 1.

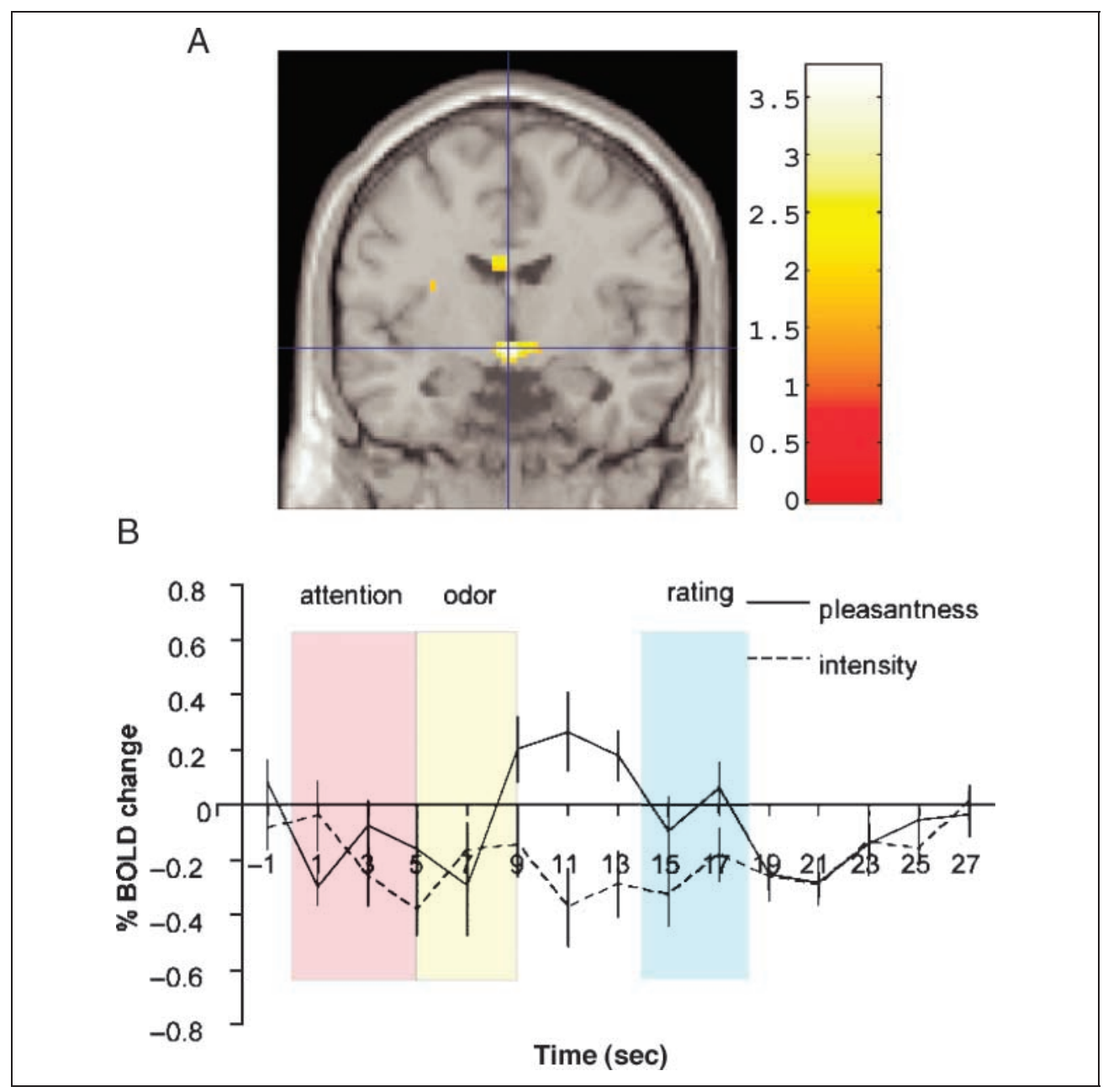


Figure 4. Odor period: effect of paying attention to intensity versus not being instructed to pay attention to intensity. (A) A significant difference during the odor period was found in the pyriform cortex at [30-2 -16], $z=3.64, p<.01$ (and also contralaterally). (B) The time courses of the activations at this location for the conditions of paying attention to intensity or to receiving jasmin without a prior instruction to pay attention to intensity (mean \pm SEM across subjects). (The peaks of the activations were significantly different: $t=3.4$, $d f=12, p=.005$.) The odor, jasmin, was identical on all trials. Conventions as in Figure 1.

\section{A}

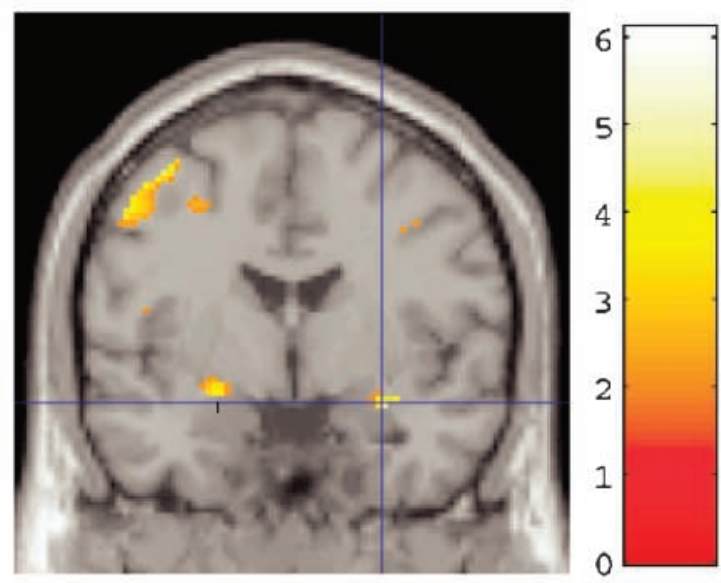

B

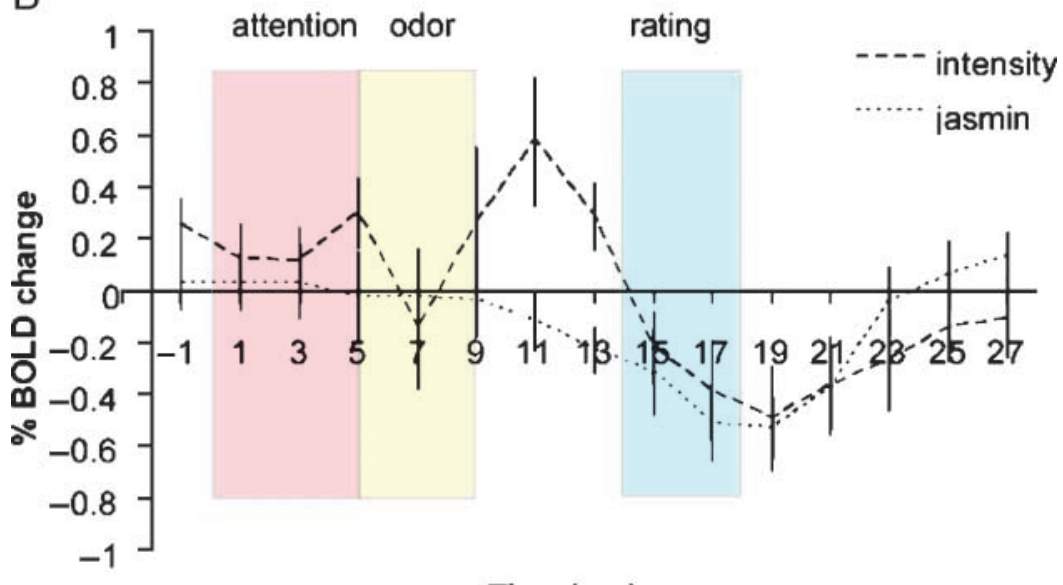

20.0\%; hexylcinnamic aldehyde, 24.0\%; hedione, 15.4\%; linalool, 24.8\%; jasmone, 4.8\%; eugenol, 2.4\%; Z3-hexenyl benzoate, $5.0 \%$; and methyl anthranilate, $3.6 \%$. The indole stimulus was $6 \%$ indole. The mixture consisted of the same concentration of all the substances in the jasmin, but in addition, $6 \%$ indole.

A purpose-designed (C. Margot) continuous-airflow six-channel computer-controlled olfactometer was used to allow odor stimuli to be delivered in the MRI scanner. The control and metal components of the system are kept outside the scanner room, and the system is free of any auditory, tactile, or thermal shifts that could cue the subject to the onset of odor delivery. The flow of cleaned medical air is controlled using a pressure regulator and a flow meter. The air is directed using solenoidoperated valves controlled by the stimulus computer using TTL pulses to one of the glass evaporation flasks containing one odor stimulus or air. The purpose of the flasks was to release an odor mixture by evaporation in a similar way to that in which an odor mixture would evaporate normally from a surface to which it had been applied. Each flask contained three filter papers held in glass flanges, on each of which was spread $0.25 \mathrm{ml}$ of the liquid odor stimulus less than $3 \mathrm{hr}$ before an experiment began. Each flask is connected by its own Teflon tube (to provide for low adhesion) to a single delivery nozzle placed within $1 \mathrm{~cm}$ of the nose to minimize dead space. The delivery nozzle provided two tubes, one for each nostril, to produce birhinal stimulation. The flow rate of the air supply was kept constant at $6 \mathrm{~L} / \mathrm{min}$. The air line was on continuously by default, and was switched off only when the solenoid directed the clean air supply to another flask so that an odorant could be delivered. This resulted in a system with no perceptible pressure change when the air was replaced during stimulus delivery by an odor for $4 \mathrm{sec}$. A similar system, though with the odorant dissolved in propylene glycol and air bubbling through the solution, was used in previous fMRI studies of human olfaction (de Araujo et al., 2005; Rolls et al., 2003).

\section{fMRI Data Acquisition}

Images were acquired with a 3.0-T Varian/Siemens whole-body scanner at the Centre for Functional Magnetic Resonance Imaging at Oxford (FMRIB), where 27 T2*-weighted echo-planar imaging (EPI) coronal slices 
with in-plane resolution of $3 \times 3 \mathrm{~mm}$ and between-plane spacing of $4 \mathrm{~mm}$ were acquired every $2 \mathrm{sec}(\mathrm{TR}=2)$. We used the techniques that we have developed over a number of years (de Araujo, Kringelbach, Rolls, \& Hobden, 2003; O'Doherty, Rolls, Francis, Bowtell, \& McGlone, 2001) and, as described in detail by Wilson et al. (2002), we carefully selected the imaging parameters in order to minimize susceptibility and distortion artifact in the orbitofrontal cortex. The relevant factors include imaging in the coronal plane, minimizing voxel size in the plane of the imaging, as high a gradient switching frequency as possible $(960 \mathrm{~Hz})$, a short echo time of $28 \mathrm{msec}$, and local shimming for the inferior frontal area. The matrix size was $64 \times 64$ and the field of view was $192 \times 192 \mathrm{~mm}$. Continuous coverage was obtained from $+62(\mathrm{~A} / \mathrm{P})$ to $-46(\mathrm{~A} / \mathrm{P})$. A whole-brain T2*-weighted EPI volume of the above dimensions and an anatomical T1 volume with coronal plane slice thickness $3 \mathrm{~mm}$ and in-plane resolution of $1 \times 1 \mathrm{~mm}$ were also acquired.

\section{fMRI Data Analysis}

The imaging data were analyzed using SPM5 (Wellcome Institute of Cognitive Neurology). Preprocessing of the data used SPM5 realignment, reslicing with sinc interpolation, normalization to the MNI coordinate system (Montreal Neurological Institute) (Collins, Neelin, Peters, \& Evans, 1994), and spatial smoothing with a 6-mm full width at half maximum isotropic Gaussian kernel. The time series at each voxel were low-pass filtered with a hemodynamic response kernel. Time-series nonsphericity at each voxel was estimated and corrected for (Friston et al., 2002), and a high-pass filter with a cutoff period of $128 \mathrm{sec}$ was applied. In the single event design, a general linear model was then applied to the time course of activation where stimulus onsets $(t=0$ in each trial) were modeled as single impulse response functions and then convolved with the canonical hemodynamic response function (Friston, Worsley, Frackowiak, Mazziotta, \& Evans, 1994). Linear contrasts were defined to test specific effects. Time derivatives were included in the basis functions set. Following smoothness estimation (Kiebel, Poline, Friston, Holmes, \& Worsley, 1999), linear contrasts of parameter estimates were defined to test the specific effects of each condition with each individual dataset. Voxel values for each contrast resulted in a statistical parametric map of the corresponding $t$ statistic, which was then transformed into the unit normal distribution (SPM $Z$ ). The statistical parametric maps from each individual dataset were then entered into second-level, random effects analyses accounting for both scan-to-scan and subject-to-subject variability. More precisely, the sets of individual statistical maps corresponding to a specific effect of interest were entered as covariates in multiple regression models as implemented in SPM5, and the corresponding group effects were assessed by applying linear contrasts (again following smoothness estimation) to the (second-level) parameter estimates generating a $t$ statistics map for each group effect of interest. The correlation analyses of the fMRI blood oxygenation level dependent (BOLD) signal with given parameters of interest (e.g., the pleasantness ratings) were performed at the second level through applying one-sample $t$ tests to the first-level statistical parametric maps resulting from performing linear parametric modulation as implemented in SPM5. We report results for brain regions where there were prior hypotheses based on olfactory activation described in previous studies, including parts of the orbitofrontal and anterior cingulate cortex, the pyriform cortex, the inferior frontal gyrus, and the hypothalamus, in which we and others have found activations in previous studies to olfactory stimuli (de Araujo et al., 2005; Anderson et al., 2003; Rolls et al., 2003; Gottfried, Deichmann, Winston, \& Dolan, 2002; Savic, Gulyas, \& Berglund, 2002; Royet et al., 2001; Zald \& Pardo, 2000) and applied smallvolume corrections for multiple comparisons (Worsley, Marrett, Neelin, Friston, \& Evans, 1996) with a radius corresponding to the full width at half maximum of the spatial smoothing filter used. Peaks are reported for which $p<.05$ in the group random effects analysis corrected for false discovery rate, although the actual corrected probability values (Worsley et al., 1996) are given in the text. All the results reported met the criterion.

To obtain the time course of the activations throughout a trial, a finite impulse response (FIR) analysis was performed as implemented in SPM, in order to make no assumption about the time course based on the temporal filtering property of the hemodynamic response function (Gottfried et al., 2006). We used 16 delta functions in the FIR analysis, spaced at intervals of the TR $(2 \mathrm{sec})$ and starting $2 \mathrm{sec}$ before the onset of the visual cue at time 0 , instructing the subject to "remember and rate pleasantness" (or intensity) on that trial. We obtained the \% BOLD signal as a function of time using the nonfitted event-related output of SPM. These time courses were obtained for statistically significant activations identified with the SPM contrast analyses that tested for a difference between paying attention to pleasantness versus intensity within the a priori regions of interest. These single-subject time courses were then averaged across subjects. Differences between the BOLD signals to the different conditions were analyzed using a $t$ test on the peak activations.

The SPM contrast analyses between the different conditions (e.g., paying attention to pleasantness vs. paying attention to intensity) started at $t=0 \mathrm{sec}$ in a trial for the attention period (the time when the visual stimulus providing instruction to remember and rate pleasantness or intensity was shown) and at $t=5 \mathrm{sec}$ for the odor period (the time when the odor delivery started). SPM reported that the contrasts were uniquely specified and were almost entirely orthogonal. The FIR analyses in SPM (which make no assumption about the 
hemodynamic response function) were performed to obtain further evidence on whether the activations reported in the article reflected activity in the attention period or the odor delivery period. The resulting FIR time-course analyses to be shown in Figures 1, 3, and 4 confirmed that different brain regions, identified by the contrast analyses as having activations related to the attention or the odor period, did have FIR activations at different times in the trial, and indeed, at approximately the time when convolution with the hemodynamic response function would predict that peaks should occur. Further evidence that the methods used in this article were able to separate activations related to the attention versus odor delivery periods is that, in some cases to be described (e.g., Figure 1 vs. Figure 3), different brain regions had differences of activations in either the attention or the odor delivery period, as indicated by both the contrast and the FIR analyses.

\section{RESULTS}

\section{Effects of Attention in the Preparatory Period before the Odor was Delivered: Pleasantness vs. Intensity}

In this period, from 0 to $5 \mathrm{sec}$ with respect to trial onset, a label was shown stating "remember and rate pleasantness" or "remember and rate intensity." Jasmin was always the odor delivered later in the trial. A contrast analysis of the pleasantness-intensity conditions showed significant differences in the medial orbitofrontal cortex (see Figure 1A) ([4 38-28], $z=2.75, p=.038)$, and in an extensive area that continued into the pregenual and subgenual cingulate cortex (see Figure 1A), with further activation peaks in the pregenual cingulate cortex ([10 $48-4], z=4.66, p \ll .001)$ and in the subgenual cingulate cortex ([ $\left.\left[\begin{array}{lll}0 & 28 & 2\end{array}\right], z=3.33, p<.02\right)$. The time courses of the activations at this medial orbitofrontal cortex location for the condition of paying attention to pleasantness or intensity are shown in Figure 1B. The BOLD response was significantly different for the pleasantness versus intensity condition by $5 \mathrm{sec}$ into the trial, reflecting effects produced by the labels which had appeared $5 \mathrm{sec}$ earlier, as this is a typical delay from stimulus onset until the peak of a BOLD response. The difference continued throughout the part of the trial in which the pleasantness or intensity had to be remembered so that a rating could be given at $t=14-18 \mathrm{sec}$. This period included the period in which the odor was delivered, at $t=5-9 \mathrm{sec}$. The time-course results for the medial orbitofrontal cortex thus indicate that being instructed to remember and rate pleasantness produces a greater activation than for intensity in the period before the odor is given, during the delivery of the odor, and in the period while the odor is being remembered $(t=9-14 \mathrm{sec})$ prior to the rating. The peaks of the activations were highly significantly different, $t=6.7$, $d f=12, p=.00002$. Similar time courses were found for other parts of the region shown in Figure 1A, including at the pregenual and subgenual cingulate cortex coordinates shown above, in which similar significant differences between the pleasantness and intensity conditions occurred in the time courses at the same 5- and 7-sec points shown in Figure 1B.

It is notable that this region of the medial orbitofrontal cortex not only had the differences of activation in the pleasantness versus intensity instruction conditions described here but also had activations in the same subjects that were correlated in the same MRI session with the subjective pleasantness ratings given on different trials to those analyzed here to the odors jasmin (pleasant), 6\% indole (unpleasant), and a mixture of jasmin and indole (e.g., with a peak at $\left[\begin{array}{lll}4 & 46 & -14\end{array}\right]$, $z=2.41, p<.05$; Grabenhorst et al., 2007). Thus, in this part of the brain where the activations are correlated with subjective pleasantness, activations are found that are related to how the odor should be processed even before the odor is delivered (with more activation if its affective value rather than its intensity should be rated), and when it must be remembered in a delay prior to being rated for pleasantness, as well as when the odor is given (Figure 1B).

The contrast for pleasantness versus intensity also showed significant differences of activation in the medial part of the head of the caudate nucleus ([ $\left[\begin{array}{lll}-6 & 12 & 10\end{array}\right]$, $z=3.37, p<.03$ ), a part of the caudate nucleus that receives in the macaque from the orbitofrontal cortex (Seleman \& Goldman-Rakic, 1985) and which contains neurons comparable in some ways with those found in the orbitofrontal cortex (Rolls, 2008). The time course was similar to that illustrated in Figure 1, with the main difference in this case being between $t=5$ and $t=7 \mathrm{sec}$, reflecting an event occurring 5 to 7 sec earlier, namely, the instruction to remember and rate pleasantness or intensity.

\section{Effects of Attention in the Preparatory Period before the Odor was Delivered: Intensity vs. Pleasantness}

A contrast analysis of the intensity-pleasantness conditions showed significant differences in the attention period in the left inferior frontal gyrus (see Figure 2A), with one peak at $[-46248], z=2.66, p<.05$. The area of contiguous voxels extended from part of area 45 anteriorly to area 44 posteriorly and included other peaks at $[-56-412], z=3.32, p<.03$ and $[-5426$ $18], z=2.98, p=.03$. The time courses of the activations at this inferior frontal gyrus location for the condition of paying attention to intensity or pleasantness are shown in Figure 2B. The BOLD response was significantly different for the intensity versus pleasantness condition by $5 \mathrm{sec}$ into the trial, reflecting effects produced by the labels which had appeared 5 sec earlier. 
The difference continued throughout the part of the trial in which the pleasantness or intensity had to be remembered so that a rating could be given at $t=14$ 18 sec. This period included the period in which the odor was delivered, at $t=5-9$ sec. The time-course results for the inferior frontal gyrus thus indicate that being instructed to remember and rate intensity produces a greater activation than being instructed to remember and rate pleasantness in the period before the odor is given, during the delivery of the odor, and in the period while the odor is being remembered $(t=$ 9-13 sec) prior to the rating.

It was of interest that activations in this area were correlated with the subjective ratings of the intensity of three odors, jasmin, 6\% indole (unpleasant), and the mixture of these two (with activation peak at $\left[\begin{array}{l}-4034 \\ 34\end{array}\right.$ $12], z=3.37, p<.03$ ) that were obtained during the same experimental run and described in more detail elsewhere (Grabenhorst et al., 2007). Thus, in an area where odor intensity is represented, the activations were modulated by instructions to remember and rate the intensity versus the pleasantness of an odor, jasmin.

This result for the inferior frontal gyrus is the converse of what was found for the medial orbitofrontal cortex, and provides evidence that the attentional and memoryrelated effects found are not just a general effect of attention, but reveal differences in activations depending on whether it is pleasantness or intensity that is relevant on a trial. The dissociation of effects shows that the effects are not due just to a nonspecific effect of an attention instruction on brain function and behavioral performance. This dissociation is supported by a twoway within-subjects ANOVA performed on the \% BOLD signals shown in Figures $1 \mathrm{~B}$ and $2 \mathrm{~B}$, which showed a significant interaction effect $[F(1,12)=8.28, p=.014]$, indicating a dissociation between the effects produced in the medial orbitofrontal cortex and in the inferior frontal gyrus by the instruction, "remember and rate pleasantness" versus "remember and rate intensity."

\section{Effects of Attention to Pleasantness during Odor Delivery}

The contrast during the odor period with onset $t=5 \mathrm{sec}$ when paying attention to pleasantness versus when paying attention to intensity showed effects in the hypothalamus as illustrated in Figure 3A. The time courses of the activations at this location for the conditions of paying attention to pleasantness or to intensity showed that there were differences that were related primarily to effects produced by the odor delivery itself, which was from $t=5$ to $t=9 \mathrm{sec}$, as shown in Figure 3B. This shows that the differences did not start during the pre-odor preparatory period. The result thus shows that paying attention to the pleasantness of an odor relative to paying attention to the intensity of an odor can produce greater effects when the odor is delivered in the hypothalamus, a brain region known to contain neurons responsive to odor (Tazawa, Onoda, \& Takagi, 1987) and the reward value of flavor (Rolls, Murzi, Yaxley, Thorpe, \& Simpson, 1986), and to be activated by olfactory stimuli in humans (Savic et al., 2002).

It was possible to extend this finding as follows in a second type of analysis. A contrast of the activations in the odor period in the instruction condition described here "remember and rate pleasantness"- the no instruction condition also revealed effects in the hypothalamus $[-2-2-6], z=3.09, p<.05$. (The "no instruction" trials were from trials in the same experiment run with only a small visual cue switched on at the start of the jasmin odor delivery, and on these trials, the participants knew that they would rate both the pleasantness and intensity of the odor later in the trial.) The finding from this contrast thus shows that there is more activation during jasmin odor delivery in the hypothalamus when there is an explicit prior instruction to rate only pleasantness, rather than when the condition was to remember both the pleasantness and the intensity. (The time course of the activations in this region for the "no instruction" trials was qualitatively similar to that for the "remember and rate intensity" trials illustrated in Figure 3B.)

The contrast in the odor period of the conditions of paying attention to the pleasantness versus intensity also showed significant effects in the medial orbitofrontal cortex as noted above, with a peak in the SPM analysis at [-4 $28-36$ ], $z=2.75, p<.05$. For the orbitofrontal cortex, in contrast to the hypothalamus, instructionrelated effects specific to pleasantness were also found prior to odor delivery, as described above.

Further, in the pregenual cingulate cortex ([ $\left.\begin{array}{llll}-6 & 50 & 8\end{array}\right]$, $z=2.37, p<.05)$ and in the medial orbitofrontal cortex ([12 54 -8], $z=3.05, p<.03$ ), activations were greater during odor delivery in the condition in which subjects had to rate both pleasantness and intensity versus the "remember and rate intensity" condition. This provides further evidence that attention to intensity leads to smaller effects in the orbitofrontal and pregenual cingulate cortex than when both pleasantness and intensity are relevant to the task. An implication is that the task instructions are important in understanding the results obtained when odors are delivered, and in olfactory neuroimaging studies.

\section{Effects of Attention to Intensity during Odor Delivery}

In the first type of analysis, the contrast during the odor period with onset $t=5 \mathrm{sec}$ when paying attention to intensity versus when paying attention to pleasantness showed effects in the olfactory tubercle (which is an area that receives directly from the olfactory tract), but the effects did not reach the adopted level of significance ([10 16-12], $z=2.78, p=.003$, uncorrected). 
However, with the second type of analysis, the contrast during the odor period with onset $t=5 \mathrm{sec}$ when paying attention to intensity versus other trials without a specific instruction to pay attention to odor, but in which the participants knew during the odor delivery period that both pleasantness and intensity had to be rated later in the trial, showed effects in the pyriform cortex as illustrated in Figure 4A ([30-2-16], $z=3.64$, $p<.01$ and $[-24-4-12], z=2.97, p<.02)$. The time courses of the activations at this location for the conditions of paying attention to intensity and the delivery of the same odor, jasmin, when both pleasantness and intensity were relevant to later ratings, showed that there were differences that were related primarily to the time of the odor delivery itself, which was from $t=5$ to $t=9 \mathrm{sec}$, as shown in Figure $4 \mathrm{~B}$ (with similar time courses in both hemispheres). Figure $4 \mathrm{~B}$ shows that the differences did not start during the pre-odor preparatory period. The result thus shows that paying attention to the intensity of an odor relative to having to rate later both the pleasantness and intensity of the same odor can produce greater effects in the pyriform cortex when the odor is delivered. The time course shows that effects of the instruction to remember and rate intensity were not found in the pyriform cortex prior to the delivery of the odor.

It is notable that this region of the pyriform cortex not only had the differences of activation in the "remember and rate intensity" versus "other trials" condition (in which no specific instruction to pay attention to only intensity or pleasantness was given), but also had activations in the same subjects that were correlated in the same MRI session with the subjective intensity ratings given on different trials to those analyzed here to the odors jasmin (pleasant), indole (unpleasant), and a mixture of jasmin and indole (e.g., with a peak at $[-18$ $4-16], z=2.31, p<.05$; Grabenhorst et al., 2007). Thus, in this part of the brain where the activations are correlated with subjective intensity (but not pleasantness), activations are found that are greater when the subject is specifically instructed to pay attention to only intensity.

The frontal gyrus also showed effects related to the instruction to pay attention to intensity versus pleasantness that were present during the odor delivery period ([42 3238$], z=2.58, p<.05$ ). Consistent with this result, significant effects were also found in the inferior frontal gyrus in a contrast for the odor delivery period for the condition "remember and rate intensity" versus trials without any such instruction in which subjects knew that they should rate both pleasantness and intensity ([44 28 6], $z=4.04, p<.005$ ).

\section{DISCUSSION}

The results show that attentional effects of how odors are processed by the brain extend far beyond whether any attention is paid or not to an odor during odor delivery, and beyond effects in the primary olfactory cortex (Zelano et al., 2005). What we have shown here is that what attribute of the stimulus attention is being paid to, its pleasantness versus intensity, can have profound influences on how different brain regions respond to the odor. Moreover, these influences can extend to periods before any odor is delivered, during the preparation period, when the brain may be preparing itself for the type of olfactory processing to be performed. For example, in the pre-odor period, when the selective attention instruction is "remember and rate pleasantness," the medial orbitofrontal cortex and the pregenual cingulate cortex become activated relative to the condition "remember and rate intensity." Part of the interpretation of this is that, given that this brain region is especially involved in affective processing (as shown by the correlation of its activations with the subjective pleasantness ratings of odors), it is becoming prepared in this pre-odor period to perform specifically affect-related olfactory processing. Moreover, this process is not just related to the expectation that an odor of a particular affective value is about to be delivered, for the same odor, jasmin, was delivered on these two types of trial. The effect is not, therefore, just a conditioned expectation of an odor, but is instead a specific effect of selective attention to prepare to process affective value, as compared to other attributes of the same stimulus, such as its intensity.

The experimental design allowed us to show that this is a selective effect of preparation for affective processing; when the instruction was to prepare for intensity processing, the activations prior to odor delivery were instead greater in another brain system, in the inferior frontal gyrus. Processing in this region has been shown to reflect short-term memory, the memory that can allow information to be stored over short periods that is usually implemented in the brain by the continuing firing of neurons (Rolls, 2008; Fuster, 2001; GoldmanRakic, 1996). The type of short-term memory in which activations of areas 44 and 45 (and nearby regions) are found is typically auditory-verbal (Gruber \& von Cramon, 2003; Wager \& Smith, 2003; Gruber, 2001). It may be that when the instruction is to remember and rate the intensity of the odor, the processing uses some type of verbal rehearsal. The interesting finding was that the activation was greater when it was intensity than pleasantness that was being remembered and rated. We propose the following hypothesis to account for this. This region, the inferior frontal gyrus including areas 44 and 45, may be the default region used for some types of short-term memory, including that for odor intensity, when the short-term memory is not specifically about affect. In contrast, when the short-term memory is about affect, the short-term memory may be implemented in a brain region that processes and represents affect, the orbitofrontal cortex (Rolls, 2005, 2008). Indeed, in this 
study, we showed that the medial orbitofrontal cortex is involved in the short-term memory for affective value, in that it maintained its activity during a short-term memory period after the odor was delivered before the rating of pleasantness was made, as shown in Figure 1B. A previous study has shown that the orbitofrontal cortex (and other frontal areas) can be activated during olfactory short-term memory (Dade, Zatorre, Evans, \& JonesGotman, 2001). The present study shows that when the affective value of an odor is in short-term memory, this activates the orbitofrontal cortex much more than when the intensity of the odor is in short-term memory.

Selective attention effects of paying attention to pleasantness versus intensity were also found during the odor delivery. Not only were these effects found in the orbitofrontal cortex (for attention to pleasantness) and in the inferior frontal gyrus (for attention to intensity), but also in the hypothalamus [which receives from the orbitofrontal cortex (Ongur \& Price, 1998), and may implement endocrine and other functions related to motivated behavior (Rolls, 2005)]. In a positron emission tomography study, Zatorre et al. (2000), consistent with the present finding, found more activation in the hypothalamus when judging the pleasantness versus the intensity of odors, although with the nature of their design, could not investigate the effects of paying attention to pleasantness versus intensity before the odor stimuli were delivered. Thus, cognitive inputs, the instructions given when they direct attention toward affect, influence not only the orbitofrontal cortex but also structures to which it projects, in order to potentially modulate the behavioral, autonomic, and endocrine effects produced in response to olfactory stimuli.

It was of interest that, in contrast, in the pyriform cortex, where activations were correlated with intensity in these same participants (Grabenhorst et al., 2007), being directed to pay attention to intensity increased activations during odor delivery compared to equally attention-demanding trials, but trials on which instead both pleasantness and intensity had to be rated. Thus, this brain area appears to be attention-dependent with respect to odor delivery in a way that reflects selective attention within the olfactory modality, with more activation to an odor, in particular, when attention is being paid to olfactory intensity versus when attention is being paid to pleasantness and intensity, as both are to be rated with no specific instruction to remember and rate intensity.

In previous research on the effects of attention on odor processing in the brain, it has been found that the pyriform cortex responds more to the presentation of an odor if a preceding task instruction indicated by a tone is for the task to detect whether an odor is present versus when the task is to press a random button independently of whether an odor has been presented (Zelano et al., 2005). In another task, there was also more activation of the pyriform cortex when the odor was delivered if the task was to rate the intensity of the odor than to rate the pitch of a tone (Zelano et al., 2005). However, these are tasks in which attention is being directed either to olfaction or not. The findings reported here extend this rather general modulatory influence to show that, given the attention being paid to the olfactory stimulus, whether the attention is to its pleasantness or intensity, can have important consequences for how a number of different brain areas involved in olfaction process the stimulus. Further, we have extended the concept by showing that, in some brain areas, the type of attention can influence the activations even before the odor is presented, reflecting a top-down preparatory influence on olfactory areas.

The top-down influence of the task instructions can be envisaged as preparing different brain regions to process in different ways the odor being delivered. The topdown influence is understood in many brain systems to operate by biased competition (Rolls, 2008; Rolls \& Deco, 2002; Desimone \& Duncan, 1995). In this biased competition model (which is now able at the integrateand-fire neuron level of implementation to account for nonlinear effects of top-down attention; Deco \& Rolls, 2005b), a relatively weak top-down input can bias how a population of neurons responds to a sensory input, and different top-down signals (instructions in the present experiment) can bias different populations of neurons so that they, rather than unbiased populations, win the competition when the stimulus appears. We hypothesize that this mechanism underlies the effects described here, with the top-down bias specifically selecting areas and neurons involved in processing affective value versus stimulus intensity. We note that with this quantitative understanding the top-down effect on neural activity is necessarily weak so that it does not dominate the sensory input (Rolls, 2008), and that therefore the top-down effects are most evident and, indeed, nonlinear in their interaction with the sensory input when the sensory input is not too strong (Deco \& Rolls, 2005b). Nevertheless, the top-down input is sufficient for it to be evident with functional neuroimaging before the test stimulus is shown (McMains et al., 2007; Giesbrecht et al., 2006; Chawla et al., 1999; Desimone \& Duncan, 1995). The present study adds to these previous findings by showing that the anticipatory effect of top-down attention operates in different processing areas depending on whether the attention is to the affective value or the intensity of the stimulus. Indeed, not only are the brain areas involved in processing the intensity or the affective value of a stimulus different as shown here and elsewhere (Rolls et al., 2003), but also the corresponding brain areas are modulated appropriately in the anticipatory period by whether attention is to be paid to affective value or intensity.

These findings are of considerable interest in relation to olfactory testing, and olfaction in general. It appears that the task instructions, or more generally, the environmental context, can influence how brain areas process 
odors with respect to affective value, and taking this into account is likely to have important implications for understanding the effects produced by sensory stimuli not only in the laboratory but also in daily life.

These findings show that when attention is paid to affective value, the brain systems engaged to prepare for, represent, and remember a sensory stimulus are different from those engaged when attention is directed to the physical properties of the identical stimulus such as its intensity. This differential biasing of brain regions engaged in processing a sensory stimulus depending on whether the cognitive demand is for affect-related versus more sensory-related processing may be an important aspect of cognition and attention.

\section{Acknowledgments}

F. G. was supported by the Gottlieb-Daimler- and Karl BenzFoundation. The investigation was performed at the Centre for Functional Magnetic Resonance Imaging of the Brain (FMRIB) at Oxford University, and we thank Peter Hobden for his help.

Reprint requests should be sent to Edmund T. Rolls, Department of Experimental Psychology, University of Oxford, South Parks Road, Oxford OX1 3UD, England, UK, or via e-mail: Edmund.Rolls@psy.ox.ac.uk; Web: www.cns.ox.ac.uk.

\section{REFERENCES}

Anderson, A. K., Christoff, K., Stappen, I., Panitz, D., Ghahremani, D. G., Glover, G., et al. (2003). Dissociated neural representations of intensity and valence in human olfaction. Nature Neuroscience, 6, 196-202.

Carmichael, S. T., Clugnet, M.-C., \& Price, J. L. (1994). Central olfactory connections in the macaque monkey. Journal of Comparative Neurology, 346, 403-434.

Chawla, D., Rees, G., \& Friston, K. J. (1999). The physiological basis of attentional modulation in extrastriate visual areas. Nature Neuroscience, 2, 671-676.

Collins, D. L., Neelin, P., Peters, T. M., \& Evans, A. C. (1994). Automatic 3D intersubject registration of MR volumetric data in standardized Talairach space. Journal of Computer Assisted Tomography, 18, 192-205.

Critchley, H. D., \& Rolls, E. T. (1996a). Hunger and satiety modify the responses of olfactory and visual neurons in the primate orbitofrontal cortex. Journal of Neurophysiology, $75,1673-1686$.

Critchley, H. D., \& Rolls, E. T. (1996b). Olfactory neuronal responses in the primate orbitofrontal cortex: Analysis in an olfactory discrimination task. Journal of Neurophysiology, 75, 1659-1672.

Dade, L. A., Zatorre, R. J., Evans, A. C., \& Jones-Gotman, M. (2001). Working memory in another dimension: Functional imaging of human olfactory working memory. Neuroimage, 14, 650-660.

de Araujo, I. E. T., Kringelbach, M. L., Rolls, E. T., \& Hobden, P. (2003). The representation of umami taste in the human brain. Journal of Neurophysiology, 90, 313-319.

de Araujo, I. E. T., Rolls, E. T., Velazco, M. I., Margot, C., \& Cayeux, I. (2005). Cognitive modulation of olfactory processing. Neuron, 46, 671-679.

Deco, G., \& Rolls, E. T. (2005a). Attention, short-term memory, and action selection: A unifying theory. Progress in Neurobiology, 76, 236-256.
Deco, G., \& Rolls, E. T. (2005b). Neurodynamics of biased competition and co-operation for attention: A model with spiking neurons. Journal of Neurophysiology, 94, 295-313.

Desimone, R., \& Duncan, J. (1995). Neural mechanisms of selective visual attention. Annual Review of Neuroscience, 18, 193-222.

Friston, K. J., Glaser, D. E., Henson, R. N., Kiebel, S., Phillips, C. \& Ashburner, J. (2002). Classical and Bayesian inference in neuroimaging: Applications. Neuroimage, 16, 484-512.

Friston, K. J., Worsley, K. J., Frackowiak, R. S. J., Mazziotta, J. C., \& Evans, A. C. (1994). Assessing the significance of focal activations using their spatial extent. Human Brain Mapping, 1, 214-220.

Fuster, J. M. (2001). The prefrontal cortex-An update: Time is of the essence. Neuron, 30, 319-333.

Giesbrecht, B., Weissman, D. H., Woldorff, M. G., \& Mangun, G. R. (2006). Pre-target activity in visual cortex predicts behavioral performance on spatial and feature attention tasks. Brain Research, 1080, 63-72.

Goldman-Rakic, P. S. (1996). The prefrontal landscape Implications of functional architecture for understanding human mentation and the central executive. Philosophical Transactions of the Royal Society of London, Series B, Biological Sciences, 351, 1445-1453.

Gottfried, J. A., Deichmann, R., Winston, J. S., \& Dolan, R. J. (2002). Functional heterogeneity in human olfactory cortex: An event-related functional magnetic resonance imaging study. Journal of Neuroscience, 22, 10819-10828.

Gottfried, J. A., Winston, J. S., \& Dolan, R. J. (2006). Dissociable codes of odor quality and odorant structure in human piriform cortex. Neuron, 49, 467-479.

Grabenhorst, F., Rolls, E. T., Margot, C., da Silva, M. A. A. P., \& Velazco, M. I. (2007). How pleasant and unpleasant stimuli combine in the brain: Odor combinations. Journal of Neuroscience, 27, 13532-13540.

Gruber, O. (2001). Effects of domain-specific interference on brain activation associated with verbal working memory task performance. Cerebral Cortex, 11, 1047-1055.

Gruber, O., \& von Cramon, D. Y. (2003). The functional neuroanatomy of human working memory revisited. Evidence from 3-T fMRI studies using classical domain-specific interference tasks. Neuroimage, 19, 797-809.

Kiebel, S. J., Poline, J. B., Friston, K. J., Holmes, A. P., \& Worsley, K. J. (1999). Robust smoothness estimation in statistical parametric maps using standardized residuals from the general linear model. Neuroimage, 10, 756-766.

Li, W., Luxenberg, E., Parrish, T., \& Gottfried, J. A. (2006). Learning to smell the roses: Experience-dependent neural plasticity in human piriform and orbitofrontal cortices. Neuron, 52, 1097-1108

McMains, S. A., Fehd, H. M., Emmanouil, T. A., \& Kastner, S. (2007). Mechanisms of feature and space-based attention: Response modulation and baseline increases. Journal of Neurophysiology, 98, 2110-2121.

O’Doherty, J., Rolls, E. T., Francis, S., Bowtell, R., \& McGlone, F. (2001). The representation of pleasant and aversive taste in the human brain. Journal of Neurophysiology, 85 , 1315-1321.

Ongur, D., \& Price, J. L. (1998). Prefrontal cortical projections to the hypothalamus in macaque monkeys. Journal of Comparative Neurology, 401, 480-505.

Ongur, D., \& Price, J. L. (2000). The organisation of networks within the orbital and medial prefrontal cortex of rats, monkeys and humans. Cerebral Cortex, 10, 206-219.

Plailly, J., Radnovich, A. J., Sabri, M., Royet, J. P., \& Kareken, D. A. (2007). Involvement of the left anterior insula and 
frontopolar gyrus in odor discrimination. Human Brain Mapping, 28, 363-372.

Poellinger, A., Thomas, R., Lio, P., Lee, A., Makris, N., Rosen, B. R., et al. (2001). Activation and habituation in olfaction-An fMRI study. Neuroimage, 13, 547-560.

Rolls, E. T. (2005). Emotion explained. Oxford: Oxford University Press.

Rolls, E. T. (2008). Memory, attention, and decision-making: A unifying computational neuroscience approach. Oxford: Oxford University Press.

Rolls, E. T., Critchley, H. D., Mason, R., \& Wakeman, E. A. (1996). Orbitofrontal cortex neurons: Role in olfactory and visual association learning. Journal of Neurophysiology, 75, 1970-1981.

Rolls, E. T., \& Deco, G. (2002). Computational neuroscience of vision. Oxford: Oxford University Press.

Rolls, E. T., Kringelbach, M. L., \& de Araujo, I. E. T. (2003). Different representations of pleasant and unpleasant odors in the human brain. European Journal of Neuroscience, 18, 695-703.

Rolls, E. T., Murzi, E., Yaxley, S., Thorpe, S. J., \& Simpson, S. J. (1986). Sensory-specific satiety: Food-specific reduction in responsiveness of ventral forebrain neurons after feeding in the monkey. Brain Research, 368, 79-86.

Royet, J. P., Hudry, J., Zald, D. H., Godinot, D., Gregoire, M. C., Lavenne, F., et al. (2001). Functional neuroanatomy of different olfactory judgments. Neuroimage, 13, 506-519.

Royet, J. P., Plailly, J., Delon-Martin, C., Kareken, D. A., \& Segebarth, C. (2003). fMRI of emotional responses to odors: Influence of hedonic valence and judgment, handedness, and gender. Neuroimage, 20, 713-728.

Savic, I., Berglund, H., Gulyas, B., \& Roland, P. (2001). Smelling of odorous sex hormone-like compounds causes sex-differentiated hypothalamic activations in humans. Neuron, 31, 661-668.

Savic, I., Gulyas, B., \& Berglund, H. (2002). Odorant differentiated pattern of cerebral activation: Comparison of acetone and vanillin. Human Brain Mapping, 17, $17-27$.

Seleman, L. D., \& Goldman-Rakic, P. S. (1985). Longitudinal topography and interdigitation of corticostriatal projections in the rhesus monkey. Journal of Neuroscience, 5, 776-794.

Small, D. M., Gerber, J. C., Mak, Y. E., \& Hummel, T. (2005). Differential neural responses evoked by orthonasal versus retronasal odorant perception in humans. Neuron, 47, 593-605.

Tazawa, Y., Onoda, N., \& Takagi, S. F. (1987). Olfactory input to the lateral hypothalamus of the old world monkey. Neuroscience Research, 4, 357-375.

Wager, T. D., \& Smith, E. E. (2003). Neuroimaging studies of working memory: A meta-analysis. Cognitive, Affective $\mathcal{E}$ Behavioral Neuroscience, 3, 255-274.

Wilson, J. L., Jenkinson, M., Araujo, I. E. T., Kringelbach, M. L., Rolls, E. T., \& Jezzard, P. (2002). Fast, fully automated global and local magnetic field optimisation for fMRI of the human brain. Neuroimage, 17, 967-976.

Winston, J. S., Gottfried, J. A., Kilner, J. M., \& Dolan, R. J. (2005). Integrated neural representations of odor intensity and affective valence in human amygdala. Journal of Neuroscience, 25, 8903-8907.

Worsley, K. J., Marrett, P., Neelin, A. C., Friston, K. J., \& Evans, A. C. (1996). A unified statistical approach for determining significant signals in images of cerebral activation. Human Brain Mapping, 4, 58-73.

Zald, D. H., \& Pardo, J. V. (2000). Functional neuroimaging of the olfactory system in humans. International Journal of Psychophysiology, 36, 165-181.

Zatorre, R. J., Jones-Gotman, M., \& Rouby, C. (2000). Neural mechanisms involved in odour pleasantness and intensity judgements. NeuroReport, 11, 2711-2716.

Zelano, C., Bensafi, M., Porter, J., Mainland, J., Johnson, B., Bremner, E., et al. (2005). Attentional modulation in human primary olfactory cortex. Nature Neuroscience, 8 , 114-120. 